\section{Comparison of higher order aberrations in eyes with aspherical or spherical intraocular lenses}

SW Kim ${ }^{1,2}, \mathrm{H} \mathrm{Ahn}^{3}$, EK Kim ${ }^{2,3}$ and T-i Kim ${ }^{2,3}$

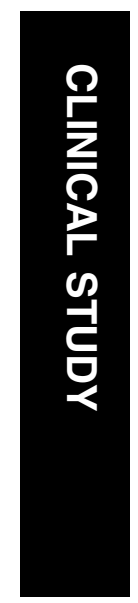

Keywords: aspheric IOL; constrast sensitivity; ocular aberration; Tecnis ZA 9003

\section{Introduction}

Over the last decade, improved surgical skills and new generation intraocular lens (IOL) designs have resulted in marked advances in cataract surgery. ${ }^{1}$ The optical quality of isolated IOLs has received considerable attention, and most IOLs have a better optical quality than crystalline lenses. However, IOLs with better optical quality does not show the same optimal quality in vivo as expected in optical laboratory system. ${ }^{1-3}$ One explanation is that optical quality is governed by both the cornea and the IOL. Studies have shown that cornea aberrations are at least partially compensated by lenticular aberrations in young human eyes. The positive spherical aberration (SA) of the cornea changes little with age. However, the lens loses its ability to compensate for aberrations of the cornea, resulting in optical quality deterioration in the aging eye. ${ }^{3}$ Optical studies show that conventional IOLs have one or two spherical surfaces, which act to increase the positive SA resulting in a blurred retinal image. ${ }^{4}$ On the basis of these observations, a new IOL was developed that converts the spherical profile to an aspherical surface to compensate for the positive SA induced by the cornea. ${ }^{5}$ The Tecnis Z9000 IOL is a 3-piece silicone foldable IOL with a prolate anterior surface introducing a negative SA to the ocular system. A number of reports verify reduced spherical aberrations associated with this IOL, and indicate that it provides superior contrast sensitivity compared to various spherical IOLs. ${ }^{6-10}$ However, there were some recent trials

\section{${ }^{1}$ Department of Ophthalmology, Soonchunhyang University College of Medicine, Bucheon, Korea}

${ }^{2}$ Department of Medicine, The Graduate School of Yonsei University, Seoul, Korea

${ }^{3}$ Department of Ophthalmology, Vision research institute, Yonsei University College of Medicine, Seoul, Korea

Correspondence: T-i Kim, Department of Ophthalmology, Yonsei University College of Medicine,

134 Shinchondong, Seodaemungu C.P.O. Box 8044 ,

Seoul 120-752,

Korea

Tel: + 8222228 3570;

Fax: + 8223120541 .

E-mail: tikim@

yuhs.ac

Received: 17 January 2008 Accepted in revised form: 18 May 2008 Published online: 17 October 2008

Informed consent was obtained from the subjects after nature of procedures had been explained No author has a financial or proprietary interest in any material or method mentioned 
showing no significant advantages in terms of subjective visual function. ${ }^{11-13}$

This study compared the optical quality of the acrylate version of the aspheric Tecnis IOL (ZA9003, AMO Inc., Santa Ana, CA, USA) with the most widely used conventional acrylate IOL (AcrySof SA60AT, Fort Worth, TX). Internal aberration (which is mostly induced by the IOL) and its contribution to the entire eye was the main interest of this study. Although most previous works used the Shack-Hartmann wavefront sensor, some limitations of that device has recently been described elsewhere. ${ }^{14}$ Notably, this study measured corneal and internal aberrations using the same apparatus, the i-Trace (Tracey Technologies, Houston, TX, USA). The i-Trace allows both wavefront and topography data to be collected, and separates corneal from internal aberrations using the ability to register with corneal topography. Thus, the relative contributions of the cornea, internal optics, and entire eye aberrations can be evaluated.

For the clinical relevance, best-corrected visual acuity (BCVA), modulation transfer function (MTF), and contrast sensitivity were assessed. Moreover, linear correlations between postoperative ocular aberrations and visual outcomes were investigated.

\section{Materials and methods}

\section{Subjects}

The study was a double-masked randomized design, and involved 50 consecutive eyes undergoing phacoemulsification for senile cataract surgery. The exclusion criteria were ocular disorders other than cataracts, systemic disease potentially affecting vision or contrast sensitivity (eg, diabetes), and intraoperative or postoperative complications including optic decentration greater than $0.5 \mathrm{~mm}$. Eligible patients were informed of the additional measures required by the study (use of wavefront aberrometer and contrast sensitivity test), and only patients who signed informed consent documents were included.

\section{Surgical procedures}

Patients were randomly assigned to the Tecnis ZA9003 or the AcrySof SA60AT groups according to uniform allocation $(1: 1)$ method. Patients were blind to which IOL they received, and surgeons were informed of the IOL at the time of surgery, and were blind to the postoperative evaluation. Surgery was performed by two experienced surgeons (TK and EK) both using the same technique. The standard surgical technique included topical anaesthesia, $3.0 \mathrm{~mm}$ temporal incision, $5.5 \mathrm{~mm}$ capsulorhexis, hydrodissection, phacoemulsification, irrigation/aspiration, and IOL implantation in the bag with the aid of the Unfolder Emerald (Tecnis) or Monarch II (AcrySof) implantation systems. Care was taken to achieve good IOL centeration and ensure a $360^{\circ}$ seal around the optic.

\section{Optical aberrations}

Wavefront aberrations were measured preoperatively and postoperatively at 3 months using the i-Trace. This device uses the fundamental principle of ray tracing, which rapidly projects thin beams of light on the retina to measure forward aberrations. The instrument allows both wavefront and corneal topography data obtained by EyeSys to be collected from the entire optical path, and can separate corneal from internal aberrations using the topographic data. An additional advantage of this approach is that the diameter of pupil analysed can be manually selected by the clinician following data collection to determine where certain aberrations occur and how they affect vision.

Pupils were dilated using Tropicamide 1\%. Wavefront maps were analysed using 4 and $6 \mathrm{~mm}$ optical zones, and higher order aberration (HOA) up to sixth Zernike coefficients were analysed. For simplicity, only the following terms were analysed: refraction parameters as given by the aberrometer (SE), $\mathrm{Z}_{3}^{-3}$ (vertical trefoil), $\mathrm{Z}_{3}^{-1}$ (vertical coma), $\mathrm{Z}_{3}^{+1}$ (horizontal coma), $\mathrm{Z}_{3}^{3}$ (horizontal trefoil), $Z_{4}^{0}$ (spherical aberration), and RMS of HOA (third to sixth order). Secondary astigmatism and quadrafoil were not considered as previous reports showed that they had little effect on visual acuity. ${ }^{9}$

\section{Visual quality test}

In addition to optical aberration data, i-Trace provides the MTF curves from 0-30 cycles/degree (cpd). We obtained MTF curves for each patient from higher order aberrations for the $6.0 \mathrm{~mm}$ optical zone. Snellen visual acuity and contrast sensitivity were evaluated with best refractive correction without pupil dilation. The operator performing the tests was not aware of the type of implanted IOL. Contrast sensitivity was measured using Optec 6500 (Stereo optical Co. Inc., Chicago, IL, USA) with the functional acuity contrast test (FACT) chart. This instrument allows testing of contrast sensitivity for distance vision under both mesopic $\left(3 \mathrm{~cd} / \mathrm{m}^{2}\right)$ and photopic $\left(85 \mathrm{~cd} / \mathrm{m}^{2}\right)$ luminance conditions. The chart displays sine wave gratings at five spatial frequencies: $1.5,3,6,12$, and $18 \mathrm{cpd}$. For each spatial frequency, the last patch of the FACT chart that was correctly read by patients before two consecutive errors was converted into the level of contrast sensitivity for that frequency, according to the conversion chart provided with the 
device. The log (base 10) of the obtained contrast sensitivity values was used for statistical analysis, and a difference level of $0.15 \log$ units between tests was considered clinically significant. 8,15

\section{Statistical analysis}

For statistical analysis, logarithm of minimum angle of resolution (logMAR) acuities and contrast sensitivity transformed into log values were used. All variables in each group were tested for normality (KolmogorovSmirnov one sample test) and homogeneity of variances (Levene test) for parametric test. Independent $t$-tests were used to compare optical qualities between two IOL groups. Simple linear regression analysis was preformed to find linear correlations between the ocular aberrations and visual outcomes. Mann-Whitney $U$-tests were used to analyse the few data that did not meet parametric criteria. Statistical analysis was performed using SPSS 12.0 software (SPSS Inc., Chicago, IL, USA), and $P$-values 0.05 or less were considered to indicate a significant difference.

\section{Results}

The postoperative clinical course was uneventful for both groups, and no case of significant IOL decentration was observed during follow-up. Of the total 50 eyes, 23 were implanted with aspheric (Tecnis ZA9003) IOLs and 25 with spherical (AcrySof SA60AT) IOLs. Two eyes implanted with aspheric IOLs were lost to follow-up. There were no statistically significant differences between the two groups in terms of age, mean IOL power, preoperative and postoperative BCVA, or postoperative refractive errors (Table 1). The three months postoperative wavefront data including total, internal, and corneal aberrations for both groups are shown in Table 2.

The mean value of aberration for each Zernike coefficient was compared between the two IOL groups. Comparison of the higher order aberration for the 4-mm pupil showed that SA differed between the two groups

Table 1 Patient characteristics for the two groups

\begin{tabular}{lccc}
\hline & Conventional & Aspheric & P-value \\
\hline Mean age & $58.8 \pm 13.5$ & $59.4 \pm 15.3$ & 0.89 \\
Number (M:F) & $25(13: 12)$ & $23(12: 11)$ & 0.99 \\
Mean IOL power (D) & $21.3 \pm 2.69$ & $21.0 \pm 1.24$ & 0.56 \\
SE at POD 3 months (D) & $-0.38 \pm 1.10$ & $-0.71 \pm 0.52$ & 0.076 \\
postoperative BCVA & $0.039 \pm 0.082$ & $0.029 \pm 0.059$ & 0.64 \\
\hline
\end{tabular}

$\mathrm{BCVA}=$ best-corrected visual acuity (logMAR); $\mathrm{POD}=$ postoperative day; $\mathrm{SE}=$ spherical equivalent.

Table 2 Postoperative total, internal, and corneal aberrations for the two IOL groups for 4.0-mm and 6.0-mm pupil diameters

\begin{tabular}{|c|c|c|c|c|c|c|}
\hline \multirow[t]{2}{*}{ Category } & \multicolumn{3}{|c|}{$4.0 \mathrm{~mm}(\mathrm{Mean} \pm S D)$} & \multicolumn{3}{|c|}{$6.0 \mathrm{~mm}($ Mean $\pm S D)$} \\
\hline & AcrySof & Tecnis & P-value & AcrySof & Tecnis & P-value \\
\hline \multicolumn{7}{|l|}{ Total } \\
\hline RMS $(\mu \mathrm{m})$ & $0.27 \pm 0.083$ & $0.34 \pm 0.22$ & 0.172 & $1.14 \pm 0.67$ & $1.30 \pm 0.93$ & 0.505 \\
\hline $\mathrm{Z}_{3}^{-1}(\mu \mathrm{m})$ & $-0.075 \pm 0.099$ & $0.033 \pm 0.14$ & $0.003^{*}$ & $-0.27 \pm 0.37$ & $-0.073 \pm 0.46$ & 0.121 \\
\hline $\mathrm{Z}_{3}^{1}(\mu \mathrm{m})$ & $0.0071 \pm 0.093$ & $0.042 \pm 0.1$ & 0.071 & $0.13 \pm 0.31$ & $0.01 \pm 0.40$ & 0.255 \\
\hline $\mathrm{Z}_{3}^{-3}(\mu \mathrm{m})$ & $-0.048 \pm 0.13$ & $-0.12 \pm 0.17$ & 0.121 & $-0.25 \pm 0.69$ & $-0.38 \pm 0.66$ & 0.505 \\
\hline $\mathrm{Z}_{3}^{3}(\mu \mathrm{m})$ & $0.0098 \pm 0.1$ & $0.064 \pm 0.14$ & 0.151 & $0.048 \pm 0.27$ & $0.10 \pm 0.46$ & 0.64 \\
\hline $\mathrm{Z}_{4}^{0}(\mu \mathrm{m})$ & $0.11 \pm 0.034$ & $-0.0061 \pm 0.058$ & $0.00^{*}$ & $0.48 \pm 0.13$ & $-0.072 \pm 0.25$ & $0.00^{*}$ \\
\hline \multicolumn{7}{|l|}{ Internal } \\
\hline RMS $(\mu \mathrm{m})$ & $0.24 \pm 0.08$ & $0.3 \pm 0.20$ & 0.178 & $0.94 \pm 0.70$ & $1.28 \pm 0.88$ & 0.173 \\
\hline $\mathrm{Z}_{3}^{-1}(\mu \mathrm{m})$ & $-0.13 \pm 0.083$ & $0.00035 \pm 0.10$ & $0.00^{*}$ & $-0.35 \pm 0.29$ & $-0.098 \pm 0.33$ & $0.012^{*}$ \\
\hline $\mathrm{Z}_{3}^{1}(\mu \mathrm{m})$ & $0.0059 \pm 0.063$ & $0.021 \pm 0.10$ & 0.529 & $0.15 \pm 0.23$ & $-0.0039 \pm 0.42$ & 0.151 \\
\hline $\mathrm{Z}_{3}^{-3}(\mu \mathrm{m})$ & $0.029 \pm 0.089$ & $-0.06 \pm 0.15$ & $0.015^{*}$ & $-0.037 \pm 0.59$ & $-0.23 \pm 0.60$ & 0.298 \\
\hline $\mathrm{Z}_{3}^{3}(\mu \mathrm{m})$ & $-0.057 \pm 0.093$ & $-0.016 \pm 0.12$ & 0.2 & $-0.16 \pm 0.27$ & $-0.15 \pm 0.43$ & 0.847 \\
\hline $\mathrm{Z}_{4}^{0}(\mu \mathrm{m})$ & $0.044 \pm 0.029$ & $-0.063 \pm 0.051$ & $0.00^{*}$ & $0.17 \pm 0.13$ & $-0.35 \pm 0.20$ & $0.00^{*}$ \\
\hline \multicolumn{7}{|l|}{ Corneal } \\
\hline RMS $(\mu \mathrm{m})$ & $0.21 \pm 0.087$ & $0.18 \pm 0.080$ & 0.34 & $0.67 \pm 0.21$ & $0.67 \pm 0.26$ & 0.978 \\
\hline $\mathrm{Z}_{3}^{-1}(\mu \mathrm{m})$ & $0.050 \pm 0.076$ & $0.033 \pm 0.073$ & 0.436 & $0.098 \pm 0.22$ & $0.025 \pm 0.31$ & 0.371 \\
\hline $\mathrm{Z}_{3}^{1}(\mu \mathrm{m})$ & $-0.013 \pm 0.066$ & $0.021 \pm 0.057$ & 0.058 & $-0.019 \pm 0.18$ & $0.014 \pm 0.19$ & 0.56 \\
\hline $\mathrm{Z}_{3}^{-3}(\mu \mathrm{m})$ & $-0.078 \pm 0.11$ & $-0.057 \pm 0.083$ & 0.474 & $-0.21 \pm 0.29$ & $-0.16 \pm 0.26$ & 0.549 \\
\hline $\mathrm{Z}_{3}^{3}(\mu \mathrm{m})$ & $0.067 \pm 0.075$ & $0.077 \pm 0.077$ & 0.646 & $0.21 \pm 0.24$ & $0.25 \pm 0.24$ & 0.58 \\
\hline $\mathrm{Z}_{4}^{0}(\mu \mathrm{m})$ & $0.069 \pm 0.025$ & $0.057 \pm 0.025$ & 0.093 & $0.31 \pm 0.076$ & $0.28 \pm 0.10$ & 0.322 \\
\hline
\end{tabular}

RMS $=$ root-mean square of higher-order aberrations (third to six order); $Z_{3}^{-1}=$ vertical coma; $Z_{3}^{+1}=$ horizontal coma; $Z_{3}^{-3}=$ trefoil $6 ; Z_{3}^{3}=$ trefoil $9 ;$ $\mathrm{Z}_{4}^{0}=$ spherical aberration; ${ }^{*} P<0.05$. 
(spherical IOL group $v s$ aspheric IOL group) in terms of ocular $(0.11 \pm 0.03$ vs $-0.006 \pm 0.1 \mu \mathrm{m}, P<0.001)$ and internal $(0.044 \pm 0.03$ vs $-0.063 \pm 0.05 \mu \mathrm{m}, P<0.001)$ aberration, but not corneal SA $(0.069 \pm 0.03$ vs $0.057 \pm 0.03 \mu \mathrm{m}, P=0.093)$. The most predominant internal aberration in the spherical group was the vertical coma $\left(\mathrm{Z}_{3}^{-1}\right)$. In contrast, the vertical coma showed negligible aberration in the aspherical group, which contributed to the two groups showing a difference in total vertical coma $(P=0.003)$. Although the two groups differed in terms of internal trefoil $\left(Z_{3}^{-3}, P=0.015\right)$, the total resulting trefoil was similar for both $(P=0.12)$. The two groups were similar in terms of other coefficients including all corneal aberrations.

Higher order aberrations measured for the $6 \mathrm{~mm}$ pupil were found to be similar to those for the $4 \mathrm{~mm}$ pupil, although the differences between the two groups in terms of SA were greater (ocular SA:0.48 \pm 0.13 vs $-0.07 \pm 0.25 \mu \mathrm{m}$, internal SA: $0.17 \pm 0.13$ vs $-0.35 \pm 0.20 \mu \mathrm{m}$, corneal SA: $0.31 \pm 0.08$ vs $0.28 \pm 0.10 \mu \mathrm{m})$. Although the internal vertical coma $\left(\mathrm{Z}_{3}^{-1}\right)$ was more negative in the spherical group, there was no statistical difference when the mean total coma was compared. Other coefficients showed no differences between the two groups.

In terms of contrast sensitivity testing, the aspheric IOL group showed clinically better results at all spatial frequencies except $1.5 \mathrm{cpd}$ under photopic condition. Statistically significant differences were observed at 3 and $6 \mathrm{cpd}$ under photopic conditions $(P=0.036,0.001$, respectively, Figure 1), and at $1.5-6 \mathrm{cpd}$ under mesopic conditions ( $P=0.001,0.002,0.005$, respectively, Figure 2 ). Measurement of MTF for the $6 \mathrm{~mm}$ pupil using i-Trace showed the aspheric group had better optical performance at 5 and $10 \mathrm{cpd}(P=0.015,0.044$, Figure 3$)$.

Correlations between BCVA, contrast sensitivity, and aberration data (RMS values of coma, trefoil, and spherical aberration) were evaluated. Total SA was found to be negatively correlated with contrast sensitivity at 6 cpd under photopic conditions $(R=-0.37, P=0.014)$, and at $1.5 \mathrm{cpd}$ under mesopic conditions $(\mathrm{R}=-0.39$, $P=0.009)$.

\section{Discussion}

A number of studies have reported on the advantages of the Tecnis Z9000 aspheric IOL in terms of ocular aberration or contrast sensitivity. ${ }^{6-10}$ This is the first report in the peer-reviewed arena on the clinical performance of the hydrophobic acrylate version of the Tecnis IOL. The study compared the Tecnis IOL with the SA60AT lens, which is one of the most favoured hydrophobic acrylic lenses. We evaluated ocular aberrations using ray-tracing technology and compared

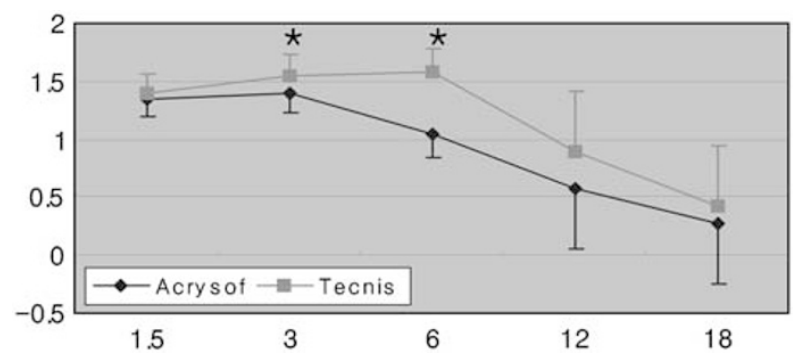

Figure 1 Mean contrast sensitivity for the two IOL groups under photopic conditions $\left(85 \mathrm{~cd} / \mathrm{m}^{2}\right) .{ }^{*} P<0.05$, error bars represent the SD.

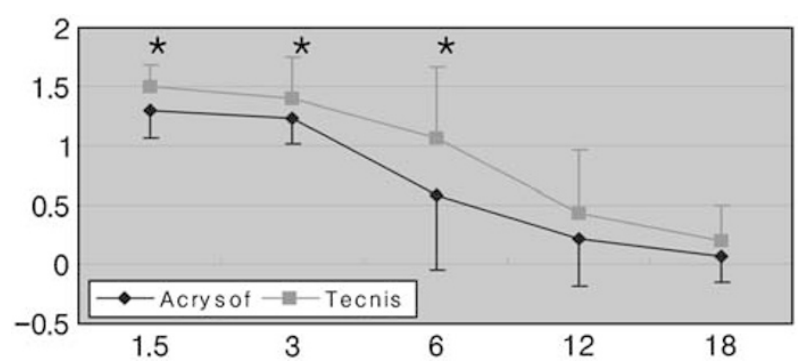

Figure 2 Mean contrast sensitivity for the two IOL groups under mesopic conditions $\left(3 \mathrm{~cd} / \mathrm{m}^{2}\right)$. ${ }^{*} P<0.05$, error bars represent the SD.

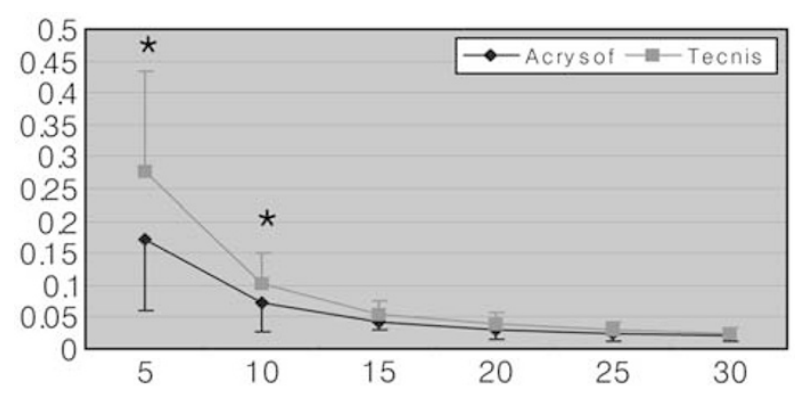

Figure 3 The MTF curve calculated from the wavefront aberration for the 6 -mm optical zone. ${ }^{*} P<0.05$, error bars represent the SD.

MTF and contrast sensitivity as an indicator of functional vision. Although the two IOLs are similar in terms of the major factors contributing to optical quality (ie, refractive index of the material and optic diameter), small differences will affect the amount of induced aberration and also the contrast sensitivity to some degree. ${ }^{8}$

A feature of this study was that it evaluated internal HOA (which is mostly induced by IOL) not merely entire eye HOA. The study examined the counterbalance effect of the corneal spherical aberration, and evaluated the magnitude of internal aberration induced by the IOL. In vivo measurement of internal aberrations allows 
assessment of the optical performance of the IOL within the eye. Therefore, knowing the corneal and lenticular aberrations can be helpful in determining whether the ocular aberration is emanating from the cornea or lens, and can thus assist in making the appropriate

adjustments to surgery, including choosing the optimum IOL. ${ }^{16}$

This study found that spherical aberrations were the greatest contributors to HOAs in the spherical IOL group, whereas they were negligible in the aspherical IOL group. It also showed that there was a considerable negative vertical coma induced primarily from the internal optic in the spherical IOL group, whereas coma induced primarily from cornea dominated in the aspheric IOL group. Although the SA and coma differed between the two groups, it did not create a difference in the RMS of total ocular HOAs. In addition, two groups differed in terms of the internal trefoil, but this did not result in a significant difference in ocular trefoil as the disparity was quite small.

The internal vertical coma was higher in the spherical IOL group than the aspheric IOL group. Marcos et $a l^{17}$ found that total vertical coma correlated with IOL vertical decentration. Although this study did not investigate minor decentration or IOL tilt in fine detail, the data suggest that the 3-piece aspheric IOL was at least as stable as the single-piece IOL.

The corneal SA is usually a positive value, thus an aspheric IOL with a negative SA value has some degree of benefit. However, in cases of a negative corneal SA (highly prolate cornea) or a positive value too low for the average, there is doubt over how much benefit there will be with an aspheric IOL designed to compensate for the average spherical aberration. Furthermore, aspheric IOL designed to compensate for the mean preoperative corneal SA can operate under the assumption that the SA remains virtually unchanged. Recent studies ${ }^{18,19}$ have shown that there are no significant changes in SA after small incision cataract surgery, and we observed similar findings (data not shown). Since surgery has little effect on corneal spherical aberration, we can theoretically use preoperative measurements to select the IOL offsetting for the corneal spherical aberration.

Several further types of aspheric IOL are now available. $^{20-22}$ Although they are theoretically similar to the Tecnis, different amounts of SA correction were introduced. The Tecnis has a lens SA of $-0.27 \mu \mathrm{m}$, aiming for zero residual SA. The IQ (Alcon, Fort Worth, TX, USA) has a lens SA of $-0.20 \mu \mathrm{m}$, targeting a residual SA of $0.1 \mu \mathrm{m}$. The Sofport AO (Bausch\&Lomb, Rochester, NY, USA) has eliminated the SA of itself, and the corneal SA of the patient remains. Although they were developed based on issues of optimal residual SA, surgeons may be able to provide more patients with better quality vision selecting adequate amounts of internal spherical aberration. This could be a possible alternative before it is available to use truly customized IOL. To this end, more study is required to establish clinical guidelines for aspheric IOL use.

Overall, the current findings indicate that the SA in patients with Tecnis IOLs was close to zero, which satisfies the aim of such lenses to compensate the corneal SA of the average patient. Moreover, the mean SA induced by the IOL was similar to $-0.27 \mu \mathrm{m}(P=0.089)$, although individual spherical aberrations were variable. However, other issues need to be addressed. First, the internal aberration was more variable than the corneal aberration. One explanation may be that the internal aberration depends not only on the IOL spherical aberration, but also on the actual position of the lens and the convergence of the rays refracted by the posterior cornea. Second, asymmetric aberrations such as, coma and trefoil, showed less consistency compared with spherical aberration. Few studies have investigated internal aberrations in vivo. The present findings are consistent with those of Belluci et $a l^{9}$ regarding the magnitude of internal aberration for the 4-mm optical zone after Tecnis Z9000 implantation. Both studies found similar mean internal spherical aberrations $(-0.048 \pm 0.017 v s-0.064 \pm 0.051 \mu \mathrm{m})$, although the fluctuations were a little higher in this study, particularly for the coma. Any differences between studies may reflect the use of different types of aberrometers. The greater variability of the present result may be because the i-Trace adapted ray-tracing technology is very flexible and robust for examining extreme aberrations, whereas Shack-Hartmann-based systems tend to have a limited dynamic range in measuring higher order aberrations. ${ }^{13}$

There is accumulating evidence supporting the functional vision benefit of aspheric over spherical IOLs, ${ }^{6-10,20-22}$ and this study is consistent with such reports. On the contrary, some recent clinical trials reported no significant gains with the aspheric IOL in terms of BCVA, ${ }^{10-13,20-22}$ or other subjective questionnaires, such as visual function-14(VF-14) score, ${ }^{13}$ or the activities of daily vision scale. ${ }^{10}$ This study also could not detect the significant difference between two IOL designs in terms of BCVA. Because of the outstanding performances of both type IOLs, Snellen visual acuity test cannot be a sufficient method to describe the quality of the vision. Subjective questionnaires also might be not enough sensitive to detect the differences between two optical design as the Moorfields IOL study group ${ }^{13}$ mentioned in their report. In fact, there is no gold standard device evaluating clinically relevant functional vision. Many studies have shown that contrast sensitivity measured under varying luminance conditions is a robust indicator of functional 
vision. ${ }^{15}$ In this study, we found that aspheric IOL group had a lower total SA that was associated with better contrast sensitivity at 3 and 6 cpd under photopic and at 1.5, 3 and $6 \mathrm{cpd}$ under mesopic conditions (Figures 1 and 2). Peak improvement was $0.47 \log$ units $(79.7 \%)$ and 0.54 $\log$ units (53.1\%) occurred at $6 \mathrm{cpd}$ under both mesopic and photopic conditions. Moreover, there was a negative correlation between the total SA and contrast sensitivity values at $1.5 \mathrm{cpd}$ under mesopic conditions and at $6 \mathrm{cpd}$ under photopic conditions.

In conclusion, this study found that the Tecnis Z9003 IOL was better than the SA60AT IOL in terms of contrast sensitivity and MTF. The total SA negatively correlated with contrast sensitivities.

\section{References}

1 Norrby NE, Grossman LW, Geraghty EP, Kreiner CF, Mihori M, Patel AS et al. Determining the imaging quality of intraocular lenses. J Cataract Refract Surg 1998; 24: 703-714.

2 Piers P, Norrby S, Mester U. Aberration-correcting intraocular lenses. In: Krueger RR, Applegate RA, MacRae SM (eds). Wavefront Customized Visual Correction: the Quest for Super Vision II. Slack: Thorofare, NJ, 2004, pp 285-290.

3 Artal P, Guirao A, Berrio E, Williams DR. Compensation of corneal aberration by the internal optics in the human eye. J Vision 2001; 1: 1-8.

4 Guirao A, Redondo M, Geraghty E, Piers P, Norrby S, Artal P. Corneal optical aberrations and retinal image quality in patients in whom monofocal intraocular lenses were implanted. Arch Ophthalmol 2002; 120: 1143-1151.

5 Holladay JT, Piers PA, Koranyi G, van der Mooren M, Norrby NE. A new intraocular lens design to reduce spherical aberration of pseudophakic eyes. J Refract Surg 2002; 18: 683-691.

6 Mester U, Dillinger P, Anterist N. Impact of a modified optic design on visual function: clinical comparative study. J Catract Refract Surg 2003; 29: 652-660.

7 Packer M, Fine IH, Hoffman RS, Piers PA. Improved functional vision with a modified prolate intraocular lens. J Refract Surg 2004; 30: 986-992.

8 Bellucci R, Scialdone A, Buratto L, Morselli S, Chierego C, Criscuoli A et al. Visual acuity and contrast sensitivity comparison between Tecnis and AcySof SA60AT intraocualr lenses: a multicenter randomized study. J Cataract Refract Surg 2005; 31: 712-717.

9 Bellucci R, Morselli S, Pucci V. Spherical aberration and coma with an aspherical and a spherical intraocular lens in normal age-matched eyes. J Cataract Refract Surg 2007; 33: 203-209.

10 Denoyer A, Lez ML, Majzoub S, Pisella P. Quality of vision after catract surgery after Tecnis Z9000 intraocular lens implantation: effect of contrast sensitivity and wavefront aberration improvements on the quality of daily vision. J Cataract Refract Surg 2007; 33: 210-216.

11 Muñoz G, Albarrán-Diego C, Montés-Micó R, Rodríguez-Galietero A, Alió JL. Spherical aberration and contrast sensitivity after cataract surgery with the Tecnis Z9000 intraocular lens. J Cataract Refract Surg 2006; 32: 1320-1327.

12 Kasper T, Bühren J, Kohnen T. Intraindividual comparison of higher-order aberration after implantation of aspheric and spherical intraocular lenses as a function of pupil diameter. J Cataract Refract Surg 2006; 32: 78-84.

13 The Moorfields IOL Study Group. Binocular implantation of the Tecnis Z9000 or AcrySof MA60AC intraocular lens in routine cataract surgery: prospective randomized controlled trial comparing VF-14 scores. J Cataract Refract Surg 2007; 33: 1559-1564.

14 Rozema JJ, Van Dyck D, Tassignon M. Clinical comparision of 6 aberrometers. Part I: technical specifications. J Cataract Refract Surg 2005; 31: 1114-1127.

15 Ginsburg AP. Contrast sensitivity: determining the visual quality and function of cataract, intraocular lenses and refractive surgery. Curr Opin Ophthalmol 2006; 17: 19-26.

16 Artal P. Combining corneal and ocular wave aberrations. In: Krueger RR, Applegate RA, MacRae SM (eds). Wavefront Customized Visual Correction: The Quest for Super Vision II. Slack: Thorofare, NJ, 2004, pp 311-316.

17 Marcos S, Rosales P, Llorente L, Jimenez-Alfaro I. Change in corneal aberrations after cataract surgery with 2 types of aspherical intraocular lenses. J Cataract Refract Surg 2007; 33: 217-226.

18 Guirao A, Tejedor J, Artal P. Corneal aberrations before and after small incision cataract surgery. Invest Ophthalmol Vis Sci 2004; 45: 4312-4319.

19 Padmanabhan P, Yoon G, Porter J, Rao S, Roy J, Choudhury M. Wavefront aberrations in eyes with AcrySof monofocal intraocular lenses. J Refract Surg 2006; 22: 237-242.

20 Rocha KM, Soriano ES, Chalita MR, Yamada AC, Bottós K, Bottós J et al. Wavefront analysis and contrast sensitivity of aspheric and spherical intraocular lenses: a randomized prospective study. Am J Ophthalmol 2006; 142: 750-756.

21 Altman GE, Nichamin Ld, Lane SS, Pepose JS. Optical performance of 3 intraocular lens designs in the presence of decentration. J Cataract Refract Surg 2005; 31: 574-585.

22 Tzelikis PF, Akaishi L, Trindade FC, Boteon JE. Ocular aberrations and contrast sensitivity after cataract surgery with AcrySof IQ intraocular lens implantation: clinical comparative study. J Cataract Refract Surg 2007; 33: 1918-1924. 